\title{
Optical coherence tomography for margin definition of basal cell carcinoma before micrographic surgery-recommendations regarding the marking and scanning technique
}

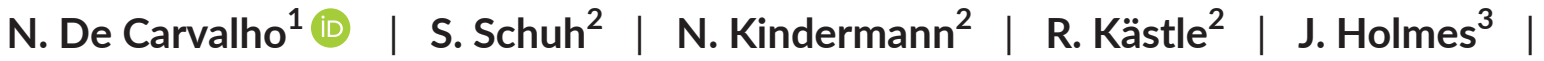 \\ J. Welzel ${ }^{2}$
}

${ }^{1}$ Department of Dermatology, University of Modena and Reggio Emilia, Modena, Italy

${ }^{2}$ Department of Dermatology, General Hospital Augsburg, Augsburg, Germany

${ }^{3}$ Michelson Diagnostics Ltd., Maidstone, UK

Correspondence

N. De Carvalho, Department of Dermatology, University of Modena and Reggio Emilia, Modena, Italy.

Email: dranathaliedecarvalho@gmail.com

\begin{abstract}
Background/purpose: Mohs Micrographic Surgery (MMS) is the preferred therapeutic treatment for high-risk basal cell carcinoma (BCC). Optical Coherence Tomography $(\mathrm{OCT})$ is a non-invasive imaging technique that enables the diagnosis of BCC. We thought to determine the margins of $B C C s$ with $\mathrm{OCT}$, prior to MMS, to reduce the number of surgical steps.
\end{abstract}

Methods: Different permanent markers were tested on the skin regarding line width, resistance against disinfection and brightness in the OCT image. The visible tumor margins of BCCs were defined by dermoscopy, adding a safety margin of $2 \mathrm{~mm}$ and labeled using the selected pen, causing a signal shadow in OCT. Scans of the center and of entire margin were performed. If parts of the BCC were visible outside the margin, another $2 \mathrm{~mm}$ were added and the scan was repeated until the tissue outside the labeling looked tumor free.

Results: Eight out of ten BCCs were totally excised in a single stage when margin delineation was done by OCT. Macroscopic margins were enlarged after OCT scanning in four patients, saving further stages of MMS.

Conclusion: OCT may help to better define the microscopic dimensions of BCCs and therefore reduce the number of stages of MMS.

\section{KEYWORDS}

basal cell carcinoma, margin mapping, Mohs micrographic surgery, non-invasive imaging, non-melanoma skin cancer, OCT, Optical coherence tomography, recurrence

\section{1 | INTRODUCTION}

Basal cell carcinoma (BCC) is the most common cancer ${ }^{1}$ and the commonest cutaneous malignancy in Caucasian. ${ }^{2}$ Its pathogenesis is correlated with chronic UV exposure and age. The incidence of new cases of BCCs is increasing. Furthermore, after the development of cutaneous carcinoma, patients have a higher risk of developing at least one more BCC in the next years. ${ }^{3,4}$ Although it is a slow-growing neoplasia and does not pose a threat to patient's life, BCCs can cause significant morbidity due to the destructive local spread and risk of recurrence if treatment is not properly conducted. ${ }^{1,3}$

According to the clinical features, BCCs are mainly classified as nodular (the most common clinical subtype, which occurs predominantly on the head), superficial (the second most common subtype, mainly located on the trunk, with a scaly appearance), and morpheaform (accounting to $5 \%-10 \%$ of the cases ${ }^{5}$ and clinically represented by a scar-like appearance). However, the final diagnosis results from the histologic findings, which classified BCCs either as nodular, 
superficial, infiltrative (corresponding to the clinically morpheaform subtype), micronodular, or basosquamous carcinoma. ${ }^{1}$

Although surgical excision is the main treatment modality for basal cell carcinomas, recurrences because of positive surgical margins are not uncommon, mainly for infiltrative, micronodular, and basosquamous carcinoma subtypes, which also tend to cause extensive local destruction and, for those reasons, are classified as "aggressive-growth subtypes". ${ }^{6}$ Besides the histologic subtype, BCCs, which are located centrofacially and periorificially (known also as the "H-zone") have a higher incidence of recurrence.

The aim of a correct treatment of BCCs relies on its complete excision with a maximum preservation of the functional capacity of the area surgically treated, along with cosmetic acceptance.

Mohs Micrographic Surgery (MMS), a specialized method of surgical procedure, is applied in the treatment of some types of skin cancers, mainly in tumors with a high-risk of relapse, recurrent BCCs and/or located in specific sites. This surgical technique is based on a minimum of two different steps. The first one consists of an excision and intraoperative microscopic confirmation of the complete margins by rapid pathology where the specimen is rightly oriented and stained aiming the detection of extra tumor foci throughout the entire border. After this procedure patients pass through other interventions, which can consist of one more surgery (closure of the defect if margins were diagnosed as free of tumor cells) or of repeated stages of further excision (until no tumor cell is detected along the margin and then the patient can finally undergo the last surgical procedure). A study made by a Spanish group specialized in MMS revealed that BCC was the most common type of skin cancer treated with MMS, consisting of almost $90 \%$ of all lesions. ${ }^{7}$ Although MMS is efficient and responsible for the highest cure rate of skin cancers ${ }^{1}$ and is the preferred therapeutic management for BCCs at high risk for recurrence, ${ }^{8,9} \mathrm{MMS}$ requires high knowledge skills of practitioners (by combining surgery with histopathology) as well as supporting histotechnicians to be able to perform this procedure, which may face some obstacles as financial and resource-related implications. ${ }^{3}$ Besides, MMS is not free of complications and patient's clinical condition may be a contraindication to the realization of this technique.

Optical Coherence Tomography (OCT) is a non-invasive imaging tool that uses a near-infrared light combining low-coherence light with interferometry to scan the skin, in real time, up to a depth of $1.8 \mathrm{~mm}$. Within this depth, the horny layer, the epidermis, the upper dermis, the skin appendages, and blood vessels are visualized. ${ }^{10}$ In crosssectional images of some millimeters length, architectural details of the upper skin layers can be assessed in a non-invasive way. ${ }^{11,12}$ OCT principles are based on the reflection of infrared radiation of the skin. Each structure of the tissue reflects a different quantity of light, resulting in scales of less or more brightness intensity of the structures evaluated.

Technical innovations of the system made the development of a multi beam OCT possible-a fast and also non-invasive scan modality. Through the reconstruction of the skin in different layers, this technique allows the evaluation of the skin in en-face/horizontal view and transversal section, the last being comparable to vertical slices of histology. Some studies have proved the capacity of OCT to diagnose $\mathrm{BCC}$ as well as to identify the subtype and estimate the depth of the neoplasia. ${ }^{13-17}$

Another application of OCT may be the pre-surgical estimation of tumor borders of BCC before MMS, which could reduce the average number of stages required for their complete excision. For these reasons, we sought for a method for margin delineation using a permanent marker and then evaluation with OCT to delimit the margins of $\mathrm{BCC}$ prior to their excision where MMS technique would be classically applied.

\section{MATERIALS AND METHODS}

As a preliminary study, lesions confirmed by histopathology as BCCs as well as those without histologic confirmation but which showed clinical and classic dermoscopic features for BCCs, were included into this pilot study. In those cases where no histologic confirmation had been obtained, OCT was used also for diagnosis of the BCC.

A total of ten BCCs underwent OCT evaluation for margin definition before patients were sent to the operating room. Lesions were located on the head and neck region: three at the nose, four in the temporal/pre-auricular region, one on the upper lip and two at the scalp. All cases were collected from the Department of Dermatology of the General Hospital of Augsburg-Germany, from October to December 2015. Institutional Review Board approval has been obtained. All patients gave their written informed consent before their inclusion into the study. Patients' age varied from 52 to 82 years, with an average of 71,5 years. Five patients were male and five were female.

All lesions were macroscopically photographed with the photo camera Sony Cyber-shot 16.1 mega pixels 5x optical zoom, and for dermoscopy evaluations we used the polarized light dermoscope Dermlite HR, 3Gen, S. Juan Capistrano, CA, USA. The VivoSight Dx OCT equipment from Michelson Diagnostics Ltd, Maidstone, Kent, UK, was used to map the BCCs. This machine carries a color video camera inside the OCT probe that guides us and allows us to know the exact part of the lesion that is evaluated. For each measurement, a "multislice" area of $6 \times 6 \mathrm{~mm}^{2}$ is scanned, comprising 120 individual cross-sectional OCT images of $6 \mathrm{~mm}$ width with an optical resolution of $7.5 \mu \mathrm{m}$ and pixel size of $4 \mu \mathrm{m}$. This "multislice" image set can be immediately reviewed frame-by-frame and also top-down ("en-face") at any desired depth.

\subsection{How we chose the material to demarcate the margin of BCC}

Most marker inks do not absorb or reflect light in the near-infrared waveband utilized by OCT and so are invisible in OCT images, making them unsuitable. We sought ink that is visible in OCT and has other attributes suitable for tumor demarcation. Four different permanent markers known for producing a hyper-reflective area upon OCT as a characteristic of their inks were systematically tested followed by comparisons among them: Edding ${ }^{\circledR} 780$ creative $0.8 \mathrm{~mm}$ in silver color, Edding ${ }^{\circledR} 1200$ metallic 54 in silver color, Edding ${ }^{\circledR} 751$ 
creative 1-2 mm in golden color and Edding ${ }^{\circledR} 780$ creative $0.8 \mathrm{~mm}$ in black color. We are also aware that white ink is also visible in OCT, such as Viscot 1440-30, but these were not evaluated in our study.

First of all to simulate the situation during surgery, an area of normal skin was disinfected with Octenisept ${ }^{\circledR}$. After the skin was naturally dried, one line with each of the pens was designed on the skin. A macroscopic picture of those lines was taken and an OCT measurement of each line was done. In the second step, the area with the lines was again disinfected with the same antiseptic product. This time Octenisept ${ }^{\circledR}$ was applied three times and the area was gently dried with a cotton swab between the disinfections. After these procedures, another macroscopic picture was taken and the lines were once more measured with OCT.

OCT images, before and after disinfection with Octenisept ${ }^{\circledR}$ for three times, were analyzed in terms of the width of the shadow in the transversal section and regarding the signal intensity below the shadow using „ImageJ” (NIH, available from http://imagej.nih.gov/ij/), respectively.

The signal intensity was measured by selecting the "histogram analysis" function of the program Image $J$ and demarcating the region of interest with the polygon tool. The histogram calculates the frequency distribution of the gray levels within the $x$-axis from 0 (corresponding to black color) to 255 (the frequency of white color) and the final average gray level determines the signal intensity of the image. To proceed with this calculation, transversal sections of OCT images of the lines made with the four different pens were selected (before and after re-disinfection), and evaluated with the histogram analysis. After those analyses, the results were compared with each other regarding the frequency distribution of the gray levels.

The permanent marker Edding ${ }^{\circledR} 780$ creative $0.8 \mathrm{~mm}$ in silver color produced the best results (Figure 1). The choice of this pen was based on the capacity of its ink to last for more than 24 hours (which was a requirement for patients who were mapped one day before surgery). Additionally, it was resistant to antiseptic products, which were used for the disinfection of the skin before starting the surgery (Octenisept ${ }^{\circledR}$ ). This pen was also preferred over the others because its tint can be visualized in the OCT image through causing a sharply demarcated strong signal shadow.

\subsection{How to acquire images and define the borders}

We developed a practical and efficient method for evaluating and adjusting the marked tumor borders with OCT. The first macroscopic demarcation of the contour of the lesions was based on the assessment with the naked eye, guided by the dermoscopic evaluation of every single lesion. On top of the visible and dermoscopic margin of the tumor, a safety distance of $2 \mathrm{~mm}$ was added, using the silver-inked pen (Figure 2). The first scan with OCT was performed in the center of the lesion. This was done for diagnostic proof as well as for the evaluation of the structural appearance of the tumor (ie, nodules, cysts). Moreover, this central scan made it possible to estimate the tumor thickness by measuring the distance from the epidermis until the inferior edge where features for $\mathrm{BCC}$ could be recognized in the transversal sections of OCT. In the next steps, OCT scans in a minimum of four different positions were carried out to measure all the margins: at 12 o'clock, at 3 o'clock, at 6 o'clock, and at 9 o'clock with a slight overlap of the $6 \times 6 \mathrm{~mm}^{2}$ area of the multislice scan (Figure 3). The flexible hand piece of the device was positioned in a way that the border demarcated with the pen was always located in the center of the probe, being visible during the whole acquisition of the images (as a guide for the mapping), so that we could exactly know which areas were measured at the moment. Thus, the scanning direction was perpendicular
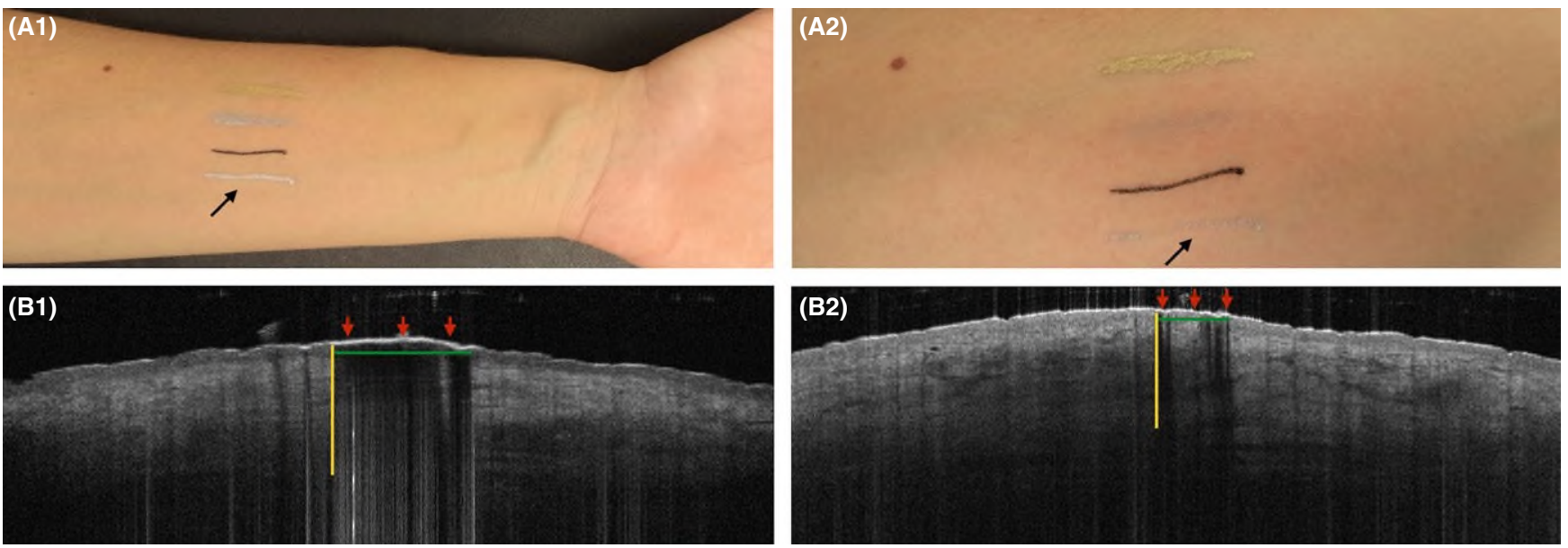

FIGURE 1 Macroscopic visualization of the lines made with the four pens tested in our study before (A1) and after (A2) three times disinfection with Octenisept ${ }^{\circledR}$. After disinfection, the ink of most of the pens is still visible (A2). The silver pen Edding ${ }^{\circledR} 780 \mathrm{creative} 0.8 \mathrm{~mm}$ is marked by black arrows. (B) Transversal section of Optical Coherence Tomography of a normal skin showing the effects of the ink of the pen Edding ${ }^{\circledR} 780$ creative $0.8 \mathrm{~mm}$ in silver color before (B1) and after (B2) three times disinfection with Octenisept ${ }^{\circledR}$. (B1) The hyper-reflective line (red arrows), the length (green horizontal line), and depth (yellow vertical line) of the shadow signal are easily identified before disinfection. Shadows are represented by well-demarcated areas with sharp lateral borders below the hyper-reflective line. (B2) After disinfection, these signs are reduced, but still visible 


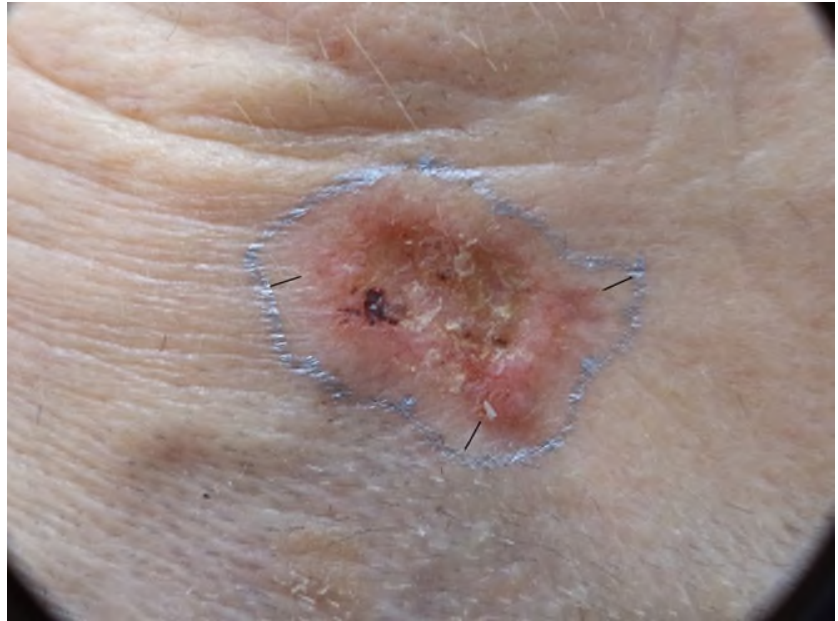

FIGURE 2 Nodular basal cell carcinoma. Delineation of the suggested surgical margin according to macroscopic and dermoscopic evaluations by keeping a distance of 2-3 $\mathrm{mm}$ from the macroscopic border of the lesion (black lines)

to the border, so that the laser could scan from the area which was dermoscopically compromised by BCC, across the pen mark to the macroscopically healthy skin. In this way, it was guaranteed that the scan covered the tumor, the dermoscopically defined margin and the area outside the border. The margin drawn with the pen appears as a hyper-reflective linear structure separating the area inside from the one outside of the demarcation and serves as a "visual guide" in the OCT image for maybe (re)-defining the border (Figure 4).

For each of the evaluations, the following OCT criteria were used for the diagnosis of BCCs, according to the "Berlin Score" system: epidermal thickening, dermal-epidermal junction (DEJ) disruption, dark border underneath epidermal thickening, hypo-reflective non-homogeneous areas in the dermis and hypo-reflective homogeneous and well defined figures in ovoid-like shapes surrounded by white stroma beneath the epidermis. ${ }^{13}$ If during the OCT scanning, any of these features was seen outside the margin, an extension of the margin of the lesion was done maintaining a distance of 2-3 mm from the previous one (Figure 5). Keeping this distance of 2-3 $\mathrm{mm}$, we followed the same length recommended by MMS technique, when it is necessary to enlarge the margins. Most of the times, the transversal sections of OCT were used for mapping, but sometimes the en-face view helped us in doubtful cases as well.

The final demarcation of the BCC borders was concluded when no suspicious area for basal cell carcinoma was visible outside the marked margin in the OCT image. At this moment, the patient was ready to undergo surgery in the following 24 hours (Figure 6).

\subsection{Histologic evaluation and correlation with OCT margin definition}

After the lesions were mapped, the surgeons chose the outermost marked margin for the excision of the lesion. The excised tissue was marked with different colors for orientation and went to the histology laboratory. There, the central part of the lesion as well as the bottom, the first margin defined by dermoscopy, all the extra margins and the

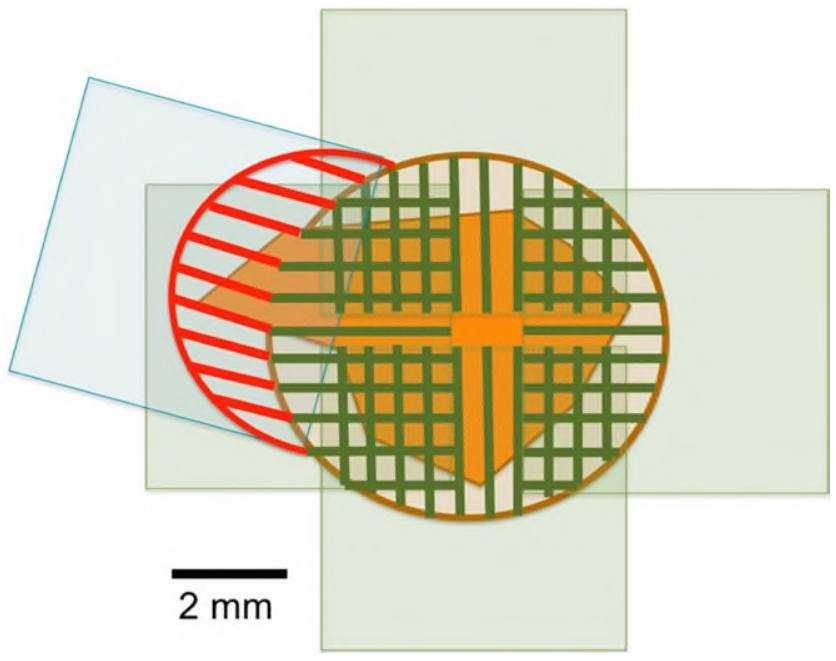

FIGURE 3 Schematic draw of how to define the margin of the lesions. A, Contour of the lesion drawn by naked eye, with the help of the dermatoscope (dark orange area in the center), and where the first OCT image is done in the central area to confirm the diagnosis of basal cell carcinoma. B, To map the border of the lesion, the probe of the device is positioned in the center of the demarcation (thin brown line surrounding the orange central area), making sure both, the lesion and the probable healthy skin, will be evaluated. The area inside the lesion (green lines inside the orange area) and the supposed healthy adjacent skin (green lines inside light green area) are measured in search for features of BCC. C, If the area outside the lesion shows features for BCC (horizontal green lines inside the light orange area), another "new margin" is drawn with the pen, keeping a distance of $2 \mathrm{~mm}$ from the first demarcation and a new image is captured with OCT (red lines). New margins are drawn and evaluated upon OCT until there is no sign for presence of BCC in the outer border of the lesion

outermost borders of the lesions that had been defined by OCT prior to MMS were evaluated separately using the "Tübinger Torte" technique. ${ }^{18}$

\section{3 | RESULTS}

\subsection{Evaluation of different markers}

According to the OCT evaluations, the best ink shadow was the one showing the narrowest length and the deepest depth, both present in pen Edding ${ }^{\circledR} 780$ creative $0.8 \mathrm{~mm}$ in silver color. Besides, also in the final macroscopic picture, taken after re-disinfections, the ink of that pen remained visible by the naked eye, allowing the surgeons to make the excisions by following the margins of the lesions determined by the OCT evaluations (Figure 1).

\subsection{Margin definition using oct and the correlation with standard histology}

The margin definition with OCT could be performed in all cases without problems. One $6 \times 6 \mathrm{~mm}^{2}$ OCT scan took 30 seconds. Together with image interpretation, the whole mapping procedure took between 15 and 20 minutes, depending on the number of additional scans for margin correction after first scanning. 


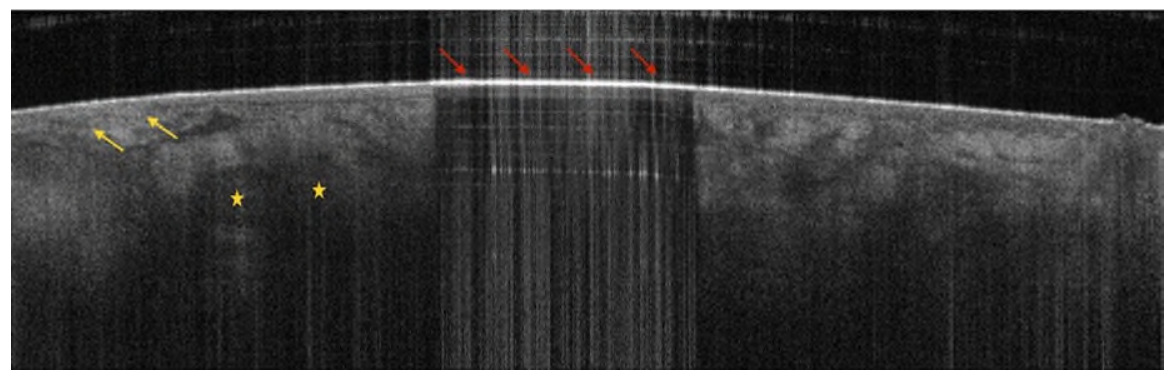

FIGURE 4 Transversal section of OCT of a basal cell carcinoma. The ink of the pen appears hyper-reflective in the center of the OCT transversal scanning (red arrows), and divides the lesion into 2 halves: inside (left side) and outside (right side) of the lesion. On the left side, there is the presence of epidermal thickening (yellow arrows), and round hypo-reflective non-homogeneous areas in the dermis surrounded by a subtle darker rim (yellow asterisks), corresponding to tumor islands of basal cell carcinoma

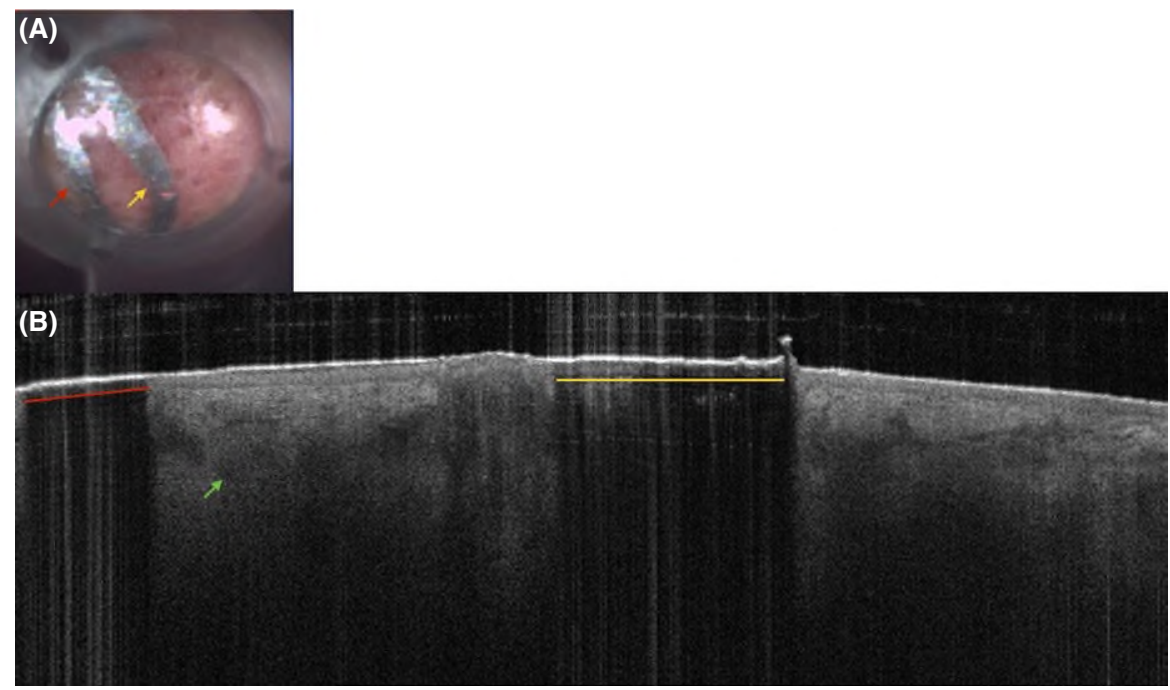

FIGURE 5 Infiltrative basal cell carcinoma. A, Image of the area that is scanned by Optical Coherence Tomography (OCT), showing two margin demarcations: the one on the left (red arrow) corresponding to the first OCT mapping and the second one, in the center of the image (yellow arrow), after OCT correction of the border. B, In the OCT evaluation, the shadows reproduced by the demarcation of the margin (A) appear as vertical dark areas below the bright sign of the pen (red and yellow lines, respectively). In the area between the two shadows, we note a subtle roundish hypo-reflective structure (green arrow) in the dermis, suggestive for a tumor island of a basal cell carcinoma. At the right side of the yellow line, no feature suggestive for BCC is seen

Concerning the histologic subtypes of the ten BCCs excised, six cases were infiltrative and four were nodular BCCs. In this first preliminary study, we had $80 \%$ of BCCs with complete excision regarding the lateral borders after margin delimitation by the OCT evaluation prior to surgery. The two BCCs, which were incompletely excised, corresponded to the infiltrative subtype (Table 1). In four other cases, the macroscopic border would have been incomplete, but the correction of the lateral margin by OCT mapping resulted in a complete excision. Regarding the lower border of the tumor, one BCC was excised incompletely. In this case, the histologic tumor thickness was $5 \mathrm{~mm}$, which is far deeper than the penetration depth of the OCT probe.

\section{DISCUSSION}

Surgery is the main treatment modality for BCCs, but recurrences because of positive surgical margins are not uncommon, mainly for the "aggressive-growth subtypes". ${ }^{6}$ Although Mohs Micrographic Surgery is the preferred treatment modality of BCCs with higher risk of recurrence, ${ }^{8,9}$ it is time-consuming, not free of complications and the patient's clinical condition may be a contraindication to the procedure of the technique.

Besides the diagnosis, OCT seems to show a high potential also for the pre-surgical assessment of the tumor, such as for the definition of the margins. In 2011 Pomerantz et al. demonstrated that OCT could be used to map tumor margins in vivo, ${ }^{19}$ but this study did not include the implementation of surgeries based on the margins mapped with the aid of OCT. This work was supplemented by Wang et al. who reported the possible benefit of using OCT to map the margins of 52 lesions before MMS. They proved, that for lesions with a single MMS, OCT margins were never larger than the eventual Mohs surgical defect, and in all cases requiring more than one Mohs stage, the OCT margins were outside the clinical estimation for the first stage. ${ }^{20}$ Alawi et al. described a technique for pre-surgical 


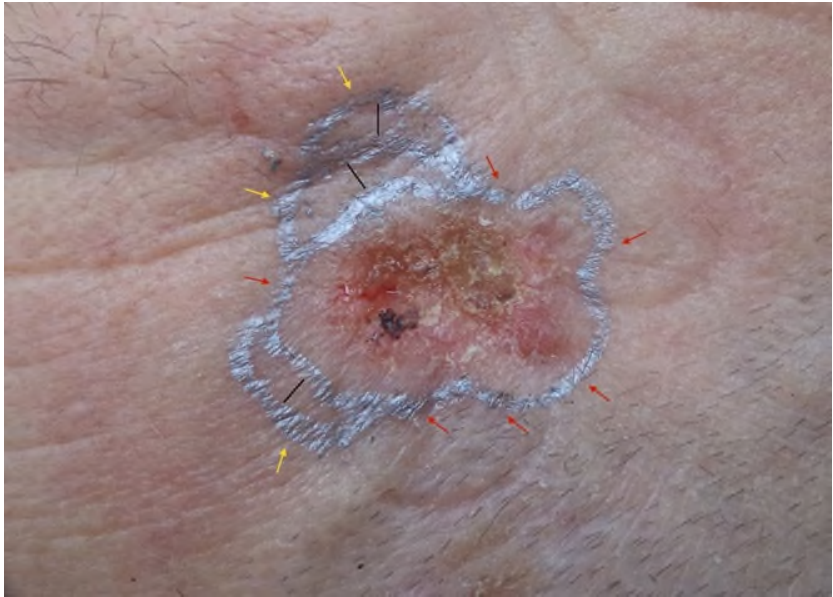

FIGURE 6 Final OCT mapping of a nodular basal cell carcinoma. Extra margins were redefined (yellow arrows) after OCT evaluation of the borders, which were previously defined through clinical and dermoscopic evaluation of the lesion (red arrows). Each extra margin was 2-3 $\mathrm{mm}$ away from the previous one (black lines)

margin assessment on non-melanocytic skin cancer by means of OCT. ${ }^{21}$ In this study, a Steri-Strip (3M Steri-Strip $6 \times 75 \mathrm{~mm}$; $3 \mathrm{M}$ $\mathrm{GmbH}$, Neuss, Germany), which gives a clear signal in OCT, was positioned in the center of the lesion as a guide to the margin definition, which was marked after OCT scanning with a waterproof pen. In this study, the surgical borders were defined according to surgeon's clinical experience, not taking into account the margin previously defined with OCT. After surgery, the OCT-defined margins were marked with tattoo or stitches on the ex vivo tumor tissue for histological analysis and comparison with the margins defined only macroscopically according to clinical experience. In all of these earlier studies, the marked OCT margins were removed prior to MMS and did not influence the surgery. Furthermore, all of these methods were timeconsuming and laborious making them potentially unsuitable for routine use.

In the present study, a pen was used to demarcate the margin of BCC, causing a signal shadow in the OCT image (Figure 4). Differently from the previous studies, in the present one the outermost margin defined by OCT was the one, which was used by the surgeons for the excisions. The procedure was developed for marking the border, scanning the entire border using OCT, evaluating the tumor lateral extent in relation to the marks and updating the marks where necessary. It was (with practice) found to be quick and efficient and we believe could be utilized in routine practice.

In cases where it was considered a re-definition of the margins due to the presence of OCT features indicative for BCC in the apparently macroscopically healthy skin, a distance of 2-3 mm was kept in order to follow the same length recommended by MMS technique, when it is necessary to enlarge the margins.

In four out of ten BCCs of this pilot study, the macroscopic/ dermoscopic margins were incorrect, missing parts of the tumors. The correction of these margins after OCT mapping resulted in a complete first step excision and saved one step of MMS for these patients. Two BCCs were incompletely excised even after OCT mapping. Both tumors were infiltrative BCCs. The most probable reason for incorrect border definition in these cases is that OCT missed small microscopic tumor islands of this ill-defined tumor subtype because of the low resolution. It should be considered that the penetration depth of OCT is limited to about $1 \mathrm{~mm}$. Therefore, OCT is only suitable to detect the superficial lateral border of the lesion and to estimate the tumor thickness in BCCs up to $1 \mathrm{~mm}$ thickness. The lower border of thicker tumors cannot be defined by this technique.

TAB LE 1 Overview of the cases of basal cell carcinomas, which were evaluated in this study. In the table, the anatomical location of the tumor, the histologic subtype, the tumor thickness, and the final histopathologic result regarding complete excision of the lateral and deep margin are given

\begin{tabular}{|llllll}
\hline Age of patient & Location & Histologic subtype & $\begin{array}{l}\text { Tumor thickness } \\
\text { upon histology }\end{array}$ & Lateral margin \\
\hline 74 & Tip of the nose & Infiltrative & $1 \mathrm{~mm}$ & Incomplete \\
\hline 66 & Nasal wing & Nodular & $0.8 \mathrm{~mm}$ & Complete & Complete \\
\hline 73 & Temporal region & Nodular & $1.1 \mathrm{~mm}$ & Complete \\
\hline 81 & Temporal region & Infiltrative & $1.5 \mathrm{~mm}$ & Incomplete \\
\hline 76 & Scalp & Infiltrative & $1.5 \mathrm{~mm}$ & Complete \\
\hline 52 & Scalp & Infiltrative & $5 \mathrm{~mm}$ & Complete \\
\hline 76 & Nodular & $1 \mathrm{~mm}$ & Macroscopic margin incomplete, \\
\hline 82 & Pre-auricular & Infiltrative & $1.45 \mathrm{~mm}$ & OCT margin complete \\
\hline 67 & Tip of the nose & Infiltrative & Macroscopic margin incomplete, \\
\hline
\end{tabular}




\section{5 | CONCLUSION}

We demonstrated a practical method by which the lateral margin of BCCs can be marked and defined through OCT evaluations, in a near to histologic evaluation of the tissue, before surgical procedures. However, imaging limitations may be bleeding tumors, crusts, infiltrative BCCs with very tiny tumor strands, and lesions positioned in difficult-to-access areas (ie, nasal wings and the auricular pavilion).

Our pilot study is limited by the small number of tumors, which underwent this procedure of pre-surgical margin mapping. The intention of our work was to develop, evaluate and describe a simple, fast and reliable method for margin definition of $\mathrm{BCC}$ relying on microscopic analysis of the tumor by means of OCT, a non-invasive imaging method.

Further work with a larger number of cases is needed, in order to quantitatively evaluate whether and how OCT reduces the excision of large safe margins and the number of stages of MMS, which are needed for reaching complete excision.

If this new method proves to statistically reduce the number of surgical stages and also reduce the average size of surgical defect, the pre-surgical margin definition of BCCs may enable an improvement of the Mohs Micrographic Surgery technique, which we may term "OCT-assisted MMS", which is more time- and resourceefficient and may enable extension of the benefits of MMS to more patients.

\section{ACKNOWLEDGEMENTS}

We would acknowledge Dr. Bernd Algermissen, Berlin, Germany, who first had the idea to use a golden Edding pen for margin definition of basal cell carcinomas using OCT.

\section{ORCID}

N. De Carvalho iD http://orcid.org/0000-0002-0823-5731

\section{REFERENCES}

1. Marzuka AG. Book SE2. Basal cell carcinoma: pathogenesis, epidemiology, clinical features, diagnosis, histopathology, and management. Yale J Biol Med. 2015;88:167-179.

2. Bath-Hextall F, Leonardi-Bee J, Smith C, et al. Trends in incidence of skin basal cell carcinoma. Additional evidence from a UK primary care database study. Int J Cancer. 2007;121:2105-2108.

3. Madan V, Lear JT, Szeimies RM. Non-melanoma skin cancer. Lancet. 2010;375:673-685.

4. Schreiber MM, Moon TE, Fox SH, Davidson J. The risk of developing subsequent nonmelanoma skin cancers. J Am Acad Dermatol. 1990;23:1114-1118.

5. Scrivener $\mathrm{Y}$, Grosshans E, Cribier B. Variations of basal cell carcinomas according to gender, age, location and histopathological subtype. $\mathrm{Br} J$ Dermatol. 2002;147:41-47.
6. Sexton M, Jones DB, Maloney ME. Histologic pattern analysis of basal cell carcinoma. Study of a series of 1039 consecutive neoplasms. J Am Acad Dermatol. 1990;23:1118-1126.

7. Ruiz-Salas V, Garcés JR, Miñano Medrano R, et al. Description of patients undergoing mohs surgery in Spain: initial report on data from the Spanish Registry of Mohs Surgery (REGESMOHS). Actas Dermosifiliogr. 2015;106:562-568.

8. Drake LA, Dinehart SM, Goltz RW, et al. Guidelines of care for Mohs micrographic surgery. American academy of dermatology. J Am Acad Dermatol. 1995;33:271-278.

9. Force AHT, Connolly SM, Baker DR, et al. AAD/ACMS/ASDSA/ASMS 2012 appropriate use criteria for Mohs micrographic surgery: a report of the American Academy of Dermatology, American College of Mohs Surgery, American Society for Dermatologic Surgery Association, and the American Society for Mohs Surgery. J Am Acad Dermatol 2012;67:531-50.. Erratu. J Am Acad Dermatol. 2015;72:748.

10. Welzel J, Lankenau E, Birngruber R, Engelhardt R. Optical coherence tomography of the human skin. J Am Acad Dermatol. 1997;37:958-963.

11. Welzel J. Optical coherence tomography. Hautarzt. 2010;61:416-420.

12. Sattler E, Kästle R, Welzel J. Optical coherence tomography in dermatology. J Biomed Opt. 2013;18:061224.

13. Wahrlich C, Alawi SA, Batz S, et al. Assessment of a scoring system for basal cell carcinoma with multi-beam optical coherence tomography. J Eur Acad Dermatol Venereol. 2015;29:1562-1569.

14. Cheng HM, Guitera P. Systematic review of optical coherence tomography usage in the diagnosis and management of basal cell carcinoma. Br J Dermatol. 2015;173:1371-1380.

15. Olmedo JM, Warschaw KE, Schmitt JM, Swanson DL. Correlation of thickness of basal cell carcinoma by optical coherence tomography in vivo and routine histologic findings: a pilot study. Dermatol Surg. 2007;33:421-425; discussion 425-6.

16. Olmedo JM, Warschaw KE, Schmitt JM, Swanson DL. Optical coherence tomography for the characterization of basal cell carcinoma in vivo: a pilot study. J Am Acad Dermatol. 2006;55:408-412.

17. Ulrich $\mathrm{M}$, von Braunmuhel $\mathrm{T}$, Kurzen $\mathrm{H}$, et al. The sensitivity and specificity of optical coherence tomography for the assisted diagnosis of nonpigmented basal cell carcinoma: an observational study. $\mathrm{Br} \mathrm{J}$ Dermatol. 2015;173:428-435.

18. Löser C, Rompel R, Breuninger H, et al. Microscopically controlled surgery (MCS). J Dtsch Dermatol Ges. 2010;8:920-925.

19. Pomerantz R, Zell D, McKenzie G, Siegel DM. Optical coherence tomography used as a modality to delineate basal cell carcinoma prior to mohs micrographic surgery. Case Rep Dermatol. 2011;3:212-218.

20. Wang KX, Meekings A, Fluhr JW, et al. Optical coherence tomographybased optimization of mohs micrographic surgery of Basal cell carcinoma: a pilot study. Dermatol Surg. 2013;39:627.

21. Alawi SA, Kuck M, Wahrlich C, et al. Optical coherence tomography for presurgical margin assessment of non-melanoma skin cancer-a practical approach. Exp Dermatol. 2013;22:547-551.

How to cite this article: De Carvalho N, Schuh S, Kindermann

N, Kästle R, Holmes J, Welzel J. Optical coherence tomography for margin definition of basal cell carcinoma before micrographic surgery-recommendations regarding the marking and scanning technique. Skin Res Technol. 2018;24:145-151. https://doi.org/10.1111/srt.12407 